\title{
Self-Assembled Three-Dimensional Deoxyribonucleic Acid (DNA) Crystals
}

\author{
Yue Zhao, ${ }^{1}$ Ruojie Sha, ${ }^{1}$ Yudong Hao, ${ }^{1}$ Carina Hernandez, ${ }^{1}$ \\ Xinshuai Zhao, ${ }^{1}$ David Rusling, ${ }^{1,}$ Jens J. Birktoft, ${ }^{1}$ Ricky Nemeth, ${ }^{2}$ \\ Christopher J. Ackerson, ${ }^{2}$ Chengde Mao $^{3}$ and Nadrian C. Seeman ${ }^{1 \star}$ \\ ${ }^{1}$ Department of Chemistry, New York University, New York, NY 10003, USA, \\ ${ }^{2}$ Department of Chemistry, Colorado State University, Fort Collins, CO 80523, USA, \\ ${ }^{3}$ Department of Chemistry, Purdue University, West Lafayette, IN 47907, USA Phone: 212- \\ 998-8395, Fax: 212-995-4475, Email: ned.seeman@nyu.edu
}

One of the major achievements of structural DNA nanotechnology is the self-assembly of 3D crystals from robust DNA motifs. One crucial goal is to use this system as a host lattice to bind guest molecules in orderly arrays for crystallography. Another goal is to control self-assembly of metallic or semiconductor nanoparticles with defined structures for creating novel electronic devices. In order to reach these two goals, we have conducted two approaches: (1) the formation and displacement of ordered DNA triplexes in self-assembled 3D DNA crystals; and (2) using gold nanoparticles modify 3D self-assembled DNA crystals.

The best diffracting crystals built to date are based on the tensegrity triangle, containing two or three double helical turns per triangular edge. An edge containing three turns of DNA is long enough to bind triplex-forming oligonucleotides (TFOs) of lengths 11 or 13 nucleotides. We have used this system as a host lattice to bind DNA triplex molecules, which are well-ordered. So as to incorporate C-containing polypyrimidine strands as triple helices, we have performed the triplex studies described here at $\mathrm{pH}$ 5.0. We have collected X-ray diffraction data to $7 \AA$ resolution on self-assembled crystals containing triplex molecules, and we have also examined the system by nondenaturing gels and by coloring the crystals with optical dyes. Our key findings are the following: [1] The TFO molecules bind in an ordered fashion to the major groove of the DNA duplexes that define the host lattice. [2] Longer TFO molecules can displace shorter TFO molecules within the crystal. [3] The Watson-Crick complement of a TFO containing a toehold will displace the TFO from its position on the duplex. [4] The TFO ceases to bind when the $\mathrm{pH}$ is raised to 7.0.

Controlled self-assembly of metallic and semiconductor nanoparticles with defined structures is an important approach for creating novel magnetic, plasmonic and photonic metamaterials. The organization of nanoparticles with DNA scaffolds in one and two dimensions have been extensively demonstrated. However, crystalline arrangement of nanoparticles into precisely designed 3D macroscopic materials for practical applications remains poorly developed. It is possible to produce a highly ordered 3D nanoparticle superlattice by using this precisely controlled well-ordered macromolecular 3D crystalline system. To demonstrate this concept, we have attached two different kinds of gold nanoclusters $(0.8 \mathrm{~nm}$ of Au25 and $1.4 \mathrm{~nm}$ of Au102) to two different DNA tensegrity triangles, which is expected to self-assemble into $3 \mathrm{D}$ macroscopic crystal with a very dense ordered arrangement of nanoparticles.

In summary, we used X-ray crystallography to visualize the TFO strands as ordered guests incorporated into the DNA lattice, and also well ordered nanoparticles.

\section{Acknowledgements}

This research has been supported by the following grants to NCS: CHE-1708776, NSF EFRI1332411 and CCF-1526650 from the NSF, Multidisciplinary University Research Initiatives (MURI) W911NF-11-1-0024 from the Army Research Office, MURI N000140911118 from the Office of Naval Research, RGP0010/2017 from Human Frontiers Science Program, DE-SC0007991 from the US Department of Energy (DOE) for DNA synthesis and partial salary support, and Grant GBMF3849 from the Gordon and Betty Moore Foundation. 